\title{
Regional variation of alanine aminotransferase serum levels in the People's Republic of China
}

\author{
Peng Li, ${ }^{1}$ Miao Ge, ${ }^{1}$ Jing Jing, ${ }^{1,2}$ Dezhi Wei ${ }^{1}$ \\ ${ }^{1}$ School of Geography and Tourism, Institute of Health Geographics, Shaanxi Normal University, Xi'an; \\ ${ }^{2}$ Baoji University of Arts and Sciences, Baoji, China
}

\begin{abstract}
The regional variation of the blood concentration of alanine aminotransferase (ALT), a sensitive predictor of liver damage, was studied in the People's Republic of China with reference to its potential association with environmental variables and geographic location. The research results presented are based on 121,977 blood samples from healthy adults in 93 cities in the country using correlation analysis, ridge regression estimation and trend surface analysis that were applied to explore if there was any tendency of spatial variation. A regression formula using a simulation equation under the condition of known local geographic factors was used. Statistical significance was set at $\mathrm{P}<0.05$. A positive correlation between ALT concentration altitude and sunshine hours and a negative correlation between ALT concentration and temperature,
\end{abstract}

Correspondence: Miao Ge, School of Geography and Tourism, Institute of Health Geographics, Shaanxi Normal University, Xi'an 710119, China.

E-mail: gmsnnu@yahoo.com

Key words: ALT distribution; Healthy adults; Ridge regression; Trend surface analysis; China.

Acknowledgements: this work was supported by the Graduate Innovation Foundation of Shaanxi Normal University and the Fundamental Research Funds for the Central Universities [grant number: 2016CSZ005]; the Graduate Innovation Foundation of Shaanxi Normal University and the Fundamental Research Funds for the Central Universities [grant number: 2016TS055]; the Central Universities Foundation of China [grant number: GK201504015]; the Graduate Innovation Foundation of Shaanxi Normal University and the Fundamental Research Funds for the Central Universities [grant number: 2016CSY012];

Conflict of interest: the authors declare no potential conflict of interest.

Received for publication: 7 March 2017.

Revision received: 1 October 2017.

Accepted for publication: 11 October 2017.

(C) Copyright P. Li et al., 2017

Licensee PAGEPress, Italy

Geospatial Health 2017; 12:472

doi:10.4081/gh.2017.472

This article is distributed under the terms of the Creative Commons Attribution Noncommercial License (CC BY-NC 4.0) which permits any noncommercial use, distribution, and reproduction in any medium, provided the original author(s) and source are credited. humidity and precipitation were found. With respect to geographical location, there was a negative correlation between ALT concentration and longitude. Higher ALT values were found in western China compared to eastern regions, dividing the country into three different regions with respect to serum ALT levels.

\section{Introduction}

The internal organs of the human body and the geographical environment in which people reside can be seen as an integrated whole, i.e. life is maintained at an equilibrium by the body obtaining what it needs from the natural environment. For example, Hippocrates, the father of western medicine, stresses that the effect of seasons should be considered in clinical medical research (Hippokrates, about $400 \mathrm{BC}$ ) and the Chinese classical work Spring and Autumn Annals (Lv, about 200 BC) reports that baldness and goitre are produced by soft water, while freshwater furthers beauty. These ancient observations acknowledge and support the idea that the functions of the body's organs are to some degree directly influenced by outside factors. Still today, some Chinese scholars are committed to the study of the effect of the environment on the body. For example, Ge et al. (2014) studied its impact on the peak expiratory flow in male children and Liu et al. (2015) discussed its effect on the $\mathrm{P}$ wave in the electrocardiogram value, while Ma et al. (2008) discovered that the height of people is affected by the geographical surroundings where they grow up.

Alanine aminotransferase (ALT) is an important enzyme found in muscles and various internal organs, such as the kidneys, heart and, most abundantly, the liver. Elevated serum ALT levels accompanies injury of these organs, and since this enzyme plays an important role in hepatic enzyme metabolism, an increased value is a strong indicator of liver damage (Xiao, 2014; Liu, 2015; Wang et al., 2015; Ekiz et al., 2016; Knudsen et al., 2016). Reviewing reported studies and published literature, we found a strong biological variability of ALT levels also without pathological organ changes, which must then be due to other factors (Drabkin, 1975; Mörl, 1983). For example, when the aerial concentration of oxygen decreases, the arterial blood oxygen also decreases and this may have an effect on the liver, which is sensitive for hypoxia (Ušaj and Burnik, 2016). Moreover, under the condition of cold temperatures and hypoxia, or other situations including low partial oxygen pressure in cold climates, regenerative cell changes (cytothesis) can decline, while increased levels of sodium and potassium ions contribute to increased cell vulnerability (Song and Wang, 2006; Wang and An, 2010) that may lead to increased release of ALT into the blood. Thus, although variations of the ALT serum level is a sensitive indicator of the liver function in clinical practice, there is also a variation of this 
enzyme with respect to variables such as sex, age, etc. that does not indicate disease. However, recognition of an association between ALT reference values and the natural environment in healthy subjects is limited. To provide a new perspective on this issue and ALT reference values, this paper explored the regional, spatial variation of ALT values in China that was studied employing ridge regression and trend surface analysis.

\section{Materials and Methods}

\section{Study area and subjects}

Measurements of serum ALT levels in a cohort of 121,977 healthy adults from 93 cities in mainland China (excluding Hong Kong, Macao and Taiwan) were collected. These sera came from 18-90 years old volunteers who were predominantly from eastern areas $(47.3 \%)$ with fewer people from middle China $(30.5 \%)$ and western parts of the country $(22.2 \%)$ due to disparities of population density. The ratio of males $(67,941)$ to females $(54,036)$ was $1: 1.3$. The subjects were all apparently healthy with the inclusion criteria being normal weight and height, i.e. body mass index (BMI) between 18.5 and $25.0 \mathrm{~kg} / \mathrm{m}^{2}$ ), normal blood pressure and normal blood glucose after 8 hours' fasting. The exclusion criteria were diagnosed, ongoing disease, such as hepatobiliary and kidney diseases (fatty liver, liver cirrhosis, hepatitis, nephritis, kidney stone, etc.), blood diseases (haemangioma, disturbance of blood circulation, other blood abnormities) or abnormal blood chemistry covering an array of different tests as used by Xue (2014). They were also subjected to abdominal ultrasonography to make sure that no hidden abnormality existed at the time of testing.

\section{Environmental variables}

Information on three classes of environmental factors were collected: spatial location and terrain indices from relevant maps and dictionaries, a meteorological index provided by the meteorological data sharing service system in China (http://www.cma.gov.cn) and a soil index obtained from the Harmonious World Soil Database (HWSD) of Vienna International Institute of Applied Systems (IIASA) supplied by the Food and Agriculture Organization (FAO) (http://www.fao.org/nr/land/ soils/harmonized-world-soil-database/zh/). In all, 22 different geographic locations and environmental variables (referred to by abbreviations $\mathrm{X}_{1}-\mathrm{X}_{22}$ ) were utilised as shown in Table 1 .

\section{Statistical approaches}

The regional variation of ALT and the potential relationship with 22 environmental variables were explored using correlation analysis (Bonett and Wright, 2000), ridge regression (Hoerl and Kennard, 1970) and trend surface analysis (Watson, 1971) while paying attention to the possibility of multiple collinearity. Student's t-test was used to the disparities of ALT values among different regions and the two sexes.

\section{Correlation analysis}

This analysis, comparing the correlation between the different datasets collected, was carried out with the Spearman method (SPSS, v. 21.0 - https://www-01.ibm.com/support/docview.wss ?uid $=$ swg21608060). P values were set at $<0.05$ for statistical significance, while $\mathrm{P}$ values $<0.01$ indicated highly significant correlations (Zar, 1972). Geographical factors constitute an organic whole as they are all connected and the statistical effectiveness can be reduced due to collinearity between the variables involved. Therefore, it was necessary to control for multicollinearity, a significant correlation between explanatory variables caused by the specific characteristics of the variables investigated that reduces the accuracy of the results (Liu and Li, 2009). This was done using SPSS v. 21.0

\section{Ridge regression analysis}

This is a regression method proposed by Hoerl and Kennard (1970) based on a biased least square method. According to the Markoff Gauss theorem (Baksalary and Kala, 1981), the regression coefficients estimation is unbiased and has a minimum variance based on the least square method under the condition of linear regression. In other words, the estimation of unbiased and minimum variance is not affected by multiple collinearity (Yang, 2004). Thus, the accuracy of this method is superior to the least square method. In this study, ridge regression was employed to explore environmental determinants on ALT values building an equation regarding ALT values and environment. The key of the ridge regression model is to confirm ridge parameter $\mathrm{K}$ (Liu and Li, 2009), the optimal solution of which depends on the unknown

Table 1. Environmental variables investigated.

\begin{tabular}{|c|c|c|c|}
\hline Variables 1-11 & Code & Variables 12-22 & Code \\
\hline Longitude & $X_{1}$ & Soil clay percentage & $\mathrm{X}_{12}$ \\
\hline Latitude & $\mathrm{X}_{2}$ & Soil reference bulk density & $\mathrm{X}_{13}$ \\
\hline Altitude & $\mathrm{X}_{3}$ & Soil bulk density & $\mathrm{X}_{14}$ \\
\hline Sunshine hours* & $X_{4}$ & Soil organic matter content & $\mathrm{X}_{15}$ \\
\hline Average temperature* & $\mathrm{X}_{5}$ & Soil $\mathrm{pH}$ value & $\mathrm{X}_{16}$ \\
\hline Average relative humidity* & $\mathrm{X}_{6}$ & Soil (clay) cation exchange capacity & $X_{17}$ \\
\hline Precipitation* & $X_{7}$ & Soil (silt) cation exchange capacity & $\mathrm{X}_{18}$ \\
\hline Temperature & $\mathrm{X}_{8}$ & Soil base saturation & $\mathrm{X}_{19}$ \\
\hline Average wind speed* & $X_{9}$ & Total exchangeable volume of soil** & $\mathrm{X}_{20}$ \\
\hline Percentage of sandy soil & $\mathrm{X}_{10}$ & Soil alkalinity & $\mathrm{X}_{21}$ \\
\hline Soil particle percentage & $X_{11}$ & Soil salinity & $\mathrm{X}_{22}$ \\
\hline
\end{tabular}

*Annual; **an indicator evaluating the capacity of the soil to interact with air, water as well as with organic and inorganic substances. 
parameters $\beta_{i}$ and $X_{i}$ in the model. The linear, analogue equation is as follows:

$$
Y=\sum_{i=1}^{n} \beta i \cdot X i+\varepsilon
$$

where $X_{i}$ and $K$ are calculated as follows:

$$
\beta(K i)=\left(X^{T} X+K i\right)^{-1} \cdot X^{T} Y
$$

where Eq. 1, $X i=\left\{X_{1} \ldots X_{n}\right\}$ presents the input variables (the 22 environmental variables in this study). $Y$ is the dependent variable (i.e. the calculated ALT values), while $\beta i=\left\{\beta_{0} \ldots \beta n\right\}$ are the parameter vectors of the function and $\varepsilon$ a residual error. In Eq. $2, \beta^{\wedge}\left(K_{i}\right)$ is a function regarding parameter $K i, i=1 \ldots \mathrm{n}$.

However, this is not the best formula to express the relation between ALT values and environmental variables, because it is difficult to assess $K$. If it tends to zero, the equation will become the same as the least squares estimate deviating from the biased estimation; however, if it tends to infinity, the ridge trace tends to zero and the equation will not have any practical significance (He and Wang, 2008). In the actual calculation, the $K$ value of the ridge trace curve tends to the ridge parameter (Yu, 2007), which means that the square error cannot be increased too much. The ridge regression estimation was simulated by the use of SAS software (http://www.statisticssolutions.com/statistical-analysis-software-sas/).

\section{Trend analysis}

The spatial variation tendency of ALT values was calculated with the statistical analysis module of the ArcGIS, v. 10.2 software (ESRI, Redlands, CA, USA) based on the simulated data from 2,322 cities and counties in China on the basis of the ridge regression equations. In this study, the spatial variation curves were plotted with longitude, latitude and ALT values a as $\mathrm{x}$-axis, $\mathrm{y}$-axis and z-axis, respectively.

A normal distribution test should be applied on the simulated dataset to select the proper method before interpolation. It is reliable that to test the normality with the K-S test method (Fasano and Franceschini, 1987). Thus, the simulated dataset was a test for normality with the SPSS software, v. 21.0, the results of which showed a $Z$ value of 10.130 at the $P<0.05$ level. These calculations inferred that the simulated dataset was not a normal distribution. Therefore, Kriging interpolation (Rathbun, 2012) should be selected. The geographical ALT distribution in healthy adults was mapped by disjunctive Kriging (Thakur et al., 2016) using the statistical analysis module ArcGIS, v 10.2.

Firstly, the simulated values were added into a layer as analysed points. Secondly, an approach of disjunctive Kriging in the statistical analysis module was employed to interpolate the unknown points. Lastly, the map of ALT distribution was extracted by the mask method. It is noteworthy that the basic layer needed to be transferred into a grid layer (http://resources.arcgis.com/zh$\mathrm{cn} / \mathrm{help} / \mathrm{main} / 10.1 /$ ).

\section{Results}

The t-test applied to the disparities of ALT values among different regions and the two sexes showed $\mathrm{P}=0.001<0.05$ and $\mathrm{P}=0.108>0.05$, respectively, which indicates there was a signifi- cant difference of ALT values between regions but not between the sexes.

\section{Correlation analysis}

As can be seen from Table 2, the condition indices were $>10$ and the Eigen-values close to zero (apart from latitude and longitude) indicating that there might exist multicollinearity between these factors (Velleman and Welsch, 1981). However, as shown in Table 3, we found a negative correlation between ALT values and environmental factors including longitude, annual average temperature, annual average relative humidity and annual precipitation, and there was a positive correlation between ALT values and both altitude and the annual sunshine hours.

Table 2. Results of the collinearity control.

\begin{tabular}{lcc} 
Variable & Eigen-value & Condition index \\
Longitude & 0.854 & 2.641 \\
Altitude & 0.145 & 6.400 \\
\hline Sunshine hours & 0.024 & 15.643 \\
Average temperature & 0.017 & 18.959 \\
\hline Average relative humidity & 0.001 & 65.018 \\
Precipitation & 0.000 & 111.011 \\
\hline
\end{tabular}

Table 3. Correlation and significance of alanine aminotransferase in healthy Chinese adults with respect to the set of environmental

\begin{tabular}{|c|c|c|c|}
\hline Variable & Code & $r$ & $\mathbf{P}$ \\
\hline Longitude & $X_{1}$ & $-0.277 * *$ & 0.000 \\
\hline Latitude & $\mathrm{X}_{2}$ & 0.025 & 0.694 \\
\hline Altitude & $\mathrm{X}_{3}$ & $0.405^{* *}$ & 0.000 \\
\hline Sunshine hours & $X_{4}$ & $0.171^{* *}$ & 0.008 \\
\hline Average temperature & $\mathrm{X}_{5}$ & $-0.192 * *$ & 0.003 \\
\hline Average relative humidity & $\mathrm{X}_{6}$ & $-0.277^{* *}$ & 0.000 \\
\hline Precipitation & $\mathrm{X}_{7}$ & $-0.175^{* *}$ & 0.006 \\
\hline Temperature & $\mathrm{X}_{8}$ & -0.085 & 0.185 \\
\hline Average wind speed & $X_{9}$ & -0.071 & 0.271 \\
\hline Percentage of sandy soil & $X_{10}$ & 0.032 & 0.822 \\
\hline Soil particle percentage & $X_{11}$ & -0.049 & 0.444 \\
\hline Soil clay percentage & $X_{12}$ & 0.018 & 0.782 \\
\hline Soil reference bulk density & $X_{13}$ & -0.012 & 0.851 \\
\hline Soil bulk density & $\mathrm{X}_{14}$ & -0.028 & 0.667 \\
\hline Soil organic matter content & $\mathrm{X}_{15}$ & 0.103 & 0.109 \\
\hline Soil pH value & $\mathrm{X}_{16}$ & -0.029 & 0.651 \\
\hline Soil (clay) cation exchange capacity & $X_{17}$ & 0.090 & 0.160 \\
\hline Soil (silt) cation exchange capacity & $\mathrm{X}_{18}$ & 0.121 & 0.059 \\
\hline Soil base saturation & $\mathrm{X}_{19}$ & -0.082 & 0.334 \\
\hline Total exchangeable volume of soil & $X_{20}$ & -0.125 & 0.052 \\
\hline Soil alkalinity & $\mathrm{X}_{21}$ & 0.068 & 0.289 \\
\hline Soil salinity & $X_{22}$ & -0.024 & 0.760 \\
\hline
\end{tabular}
variables investigated.

The correlation coefficient is indicated by $r(r>0$ indicates a positive correlation; and $r<0$ indicates a negative correlation). ${ }^{*}$ Strong statistical significance. 


\section{Ridge regression}

Table 4 shows that the variance inflation factor (VIF) of each coefficient was $<10$ in our study. When the ridge parameter $K$ increased by 0.1 , following the tendency of the ridge trace to gradually stabilise, the mean square error (MSE) also increased leading to a simultaneous decrease in accuracy. Compared with the least square method, the MSE only increased by 0.08201 . Considering this, the ridge parameter $K=0.3$ was selected since the ridge trace tended to be stable at this value.

The ridge traces of ALT for the healthy adults are presented in Figure 1, where the variation of the $K$ parameter is shown and the estimated coefficients read. When the ridge parameter $K$ equalled 0.3 , the MSE amounted to 6.90123 (Table 4) and at that $K$-level, the constant becomes 30.4476 (Table 5) and the coefficients $0.05713,0.00230,0.00702,0.00618,-0.04830,0.00026$ of $X_{1}, X_{2}$, $\mathrm{X}_{3}, \mathrm{X}_{4}, \mathrm{X}_{5}$ and $\mathrm{X}_{6}$, respectively. The ALT equation is the following:
$\mathrm{Y}=30.45-0.05713 \mathrm{X}_{1}+0.002300 \mathrm{X}_{2}+0.007020 \mathrm{X} 3+0.006180 \mathrm{X}_{4}$ $-0.04830 \mathrm{X}_{5}+0.0002600 \mathrm{X}_{6} \pm 6.901$

Eq. 3

From Eq. 3, the actual ALT of local, healthy adults can be calculated in relation to the geographical variables under study. The simulated values and true values were tested by the paired t-test using SPSS, v. 21.0 (Zhu and He, 2009) (Table 6). The results designated by $\mathrm{P}=0.127>0.05$ are considered not to be significant with $95 \%$ confidence intervals between the true and simulated values. Put another way, the simulated values can be used as reference.

\section{Trend analysis}

As presented in Figure 2, the ALT values for healthy adults along the longitude showed a decreasing trend from west to east (the red curve), while it first increased from south to north along the latitude and then decreased slightly in the extreme North (the

Table 4. The ridge parameters.

\begin{tabular}{|c|c|c|c|c|c|c|c|}
\hline K-value & MSE & $\mathrm{X}_{1}$ & $\mathrm{X}_{2}$ & $\mathbf{X}_{3}$ & $\mathrm{X}_{4}$ & $\mathbf{X}_{5}$ & $\mathbf{X}_{6}$ \\
\hline 0.0 & 6.81922 & 5.16801 & 5.63270 & 3.68974 & 5.07754 & 6.29636 & 3.97702 \\
\hline 0.1 & 6.85304 & 1.30908 & 1.63045 & 1.33548 & 1.66241 & 1.83292 & 1.62458 \\
\hline 0.2 & 6.88086 & 0.72647 & 0.86383 & 0.83947 & 0.91555 & 0.92751 & 0.94244 \\
\hline 0.3 & 6.90123 & 0.51061 & 0.56371 & 0.61156 & 0.60512 & 0.57764 & 0.63726 \\
\hline 0.4 & 6.91763 & 0.39823 & 0.41016 & 0.47708 & 0.44055 & 0.40301 & 0.46955 \\
\hline 0.5 & 6.93169 & 0.32821 & 0.31894 & 0.38765 & 0.34068 & 0.30240 & 0.38555 \\
\hline 0.6 & 6.94421 & 0.27962 & 0.25923 & 0.32387 & 0.27457 & 0.23847 & 0.29568 \\
\hline 0.7 & 6.95524 & 0.24346 & 0.21739 & 0.27618 & 0.22804 & 0.19502 & 0.24601 \\
\hline 0.8 & 6.96625 & 0.21527 & 0.18655 & 0.23929 & 0.19378 & 0.16393 & 0.20915 \\
\hline 0.9 & 6.97621 & 0.19254 & 0.16292 & 0.21000 & 0.16764 & 0.14077 & 0.18089 \\
\hline 1.0 & 6.98564 & 0.17376 & 0.14426 & 0.18624 & 0.14713 & 0.12296 & 0.15863 \\
\hline
\end{tabular}

MSE, mean square error; VIF, variance inflation factor.

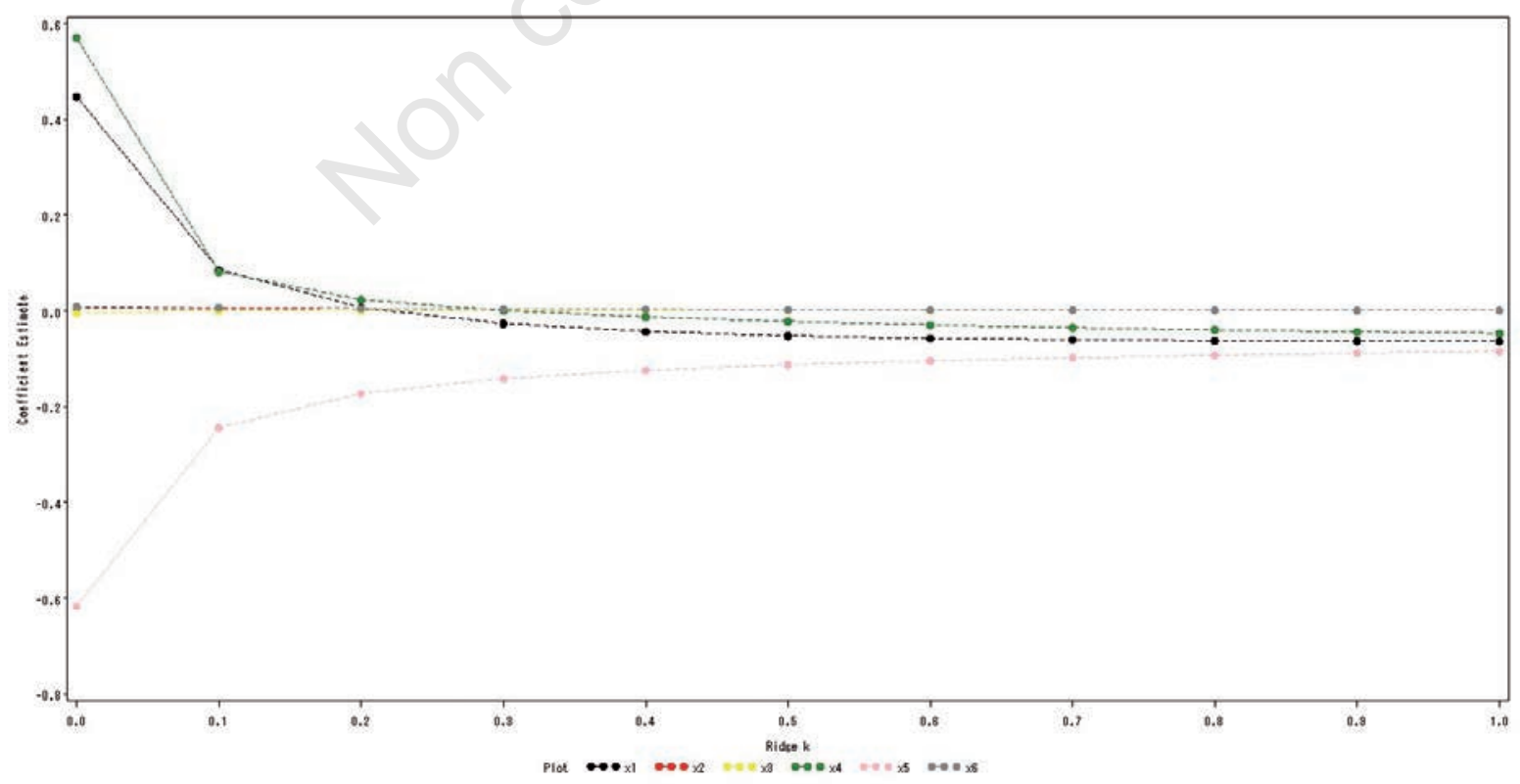

Figure 1. The ridge traces of alanine aminotransferase for healthy Chinese adults. The $\mathrm{X}_{1}-\mathrm{X}_{6}$ lines signify the variation trends of the $\mathrm{K}$ parameter at different levels of the estimated equation coefficients. 
blue curve). Although the two curves reflect the change of ALT values along purely geographic directions, the topographic map of China reveals that they also changed with respect to altitude. With the ordinate ( $\mathrm{x}$ axis) depicting longitude, the abscissa (y direction) latitude and Z the ALT concentration, it can be seen that the ALT values decrease in the eastern direction (the red slope), while they first increase in the northern direction along the latitude and then decreased slightly at the end (the blue curve).

The ALT values in healthy adults were found to be affected by the environment as governed by geographic location. The ALT values in healthy adults varied from 20.24 to 41.24 units per litre (U/L) serum. The average rate of ALT values in south-western regions (red areas on the map) varied between 34.24 and 41.24 U/L serum, while the rate of average ALT values (orange areas on the map) varied from 27.24 to $37.24 \mathrm{U} / \mathrm{L}$ serum. The values in the remaining areas (green on the map) varied from 20.24 to 27.24 U/L. Thus, the highest values were mainly found on the Tibetan Plateau, while the eastern plains and western regions had lower values (Figure 3).

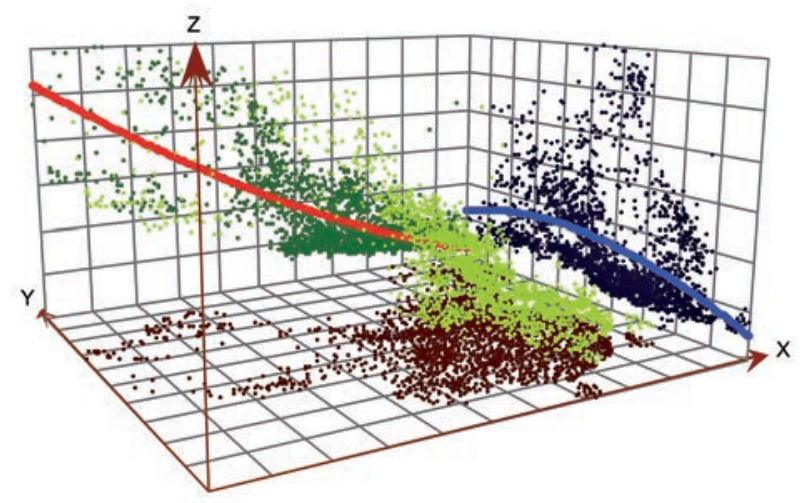

Figure 2. Variation of alanine aminotransferase values for healthy Chinese adults along geographic directions.

Table 5. The regression coefficients.

\begin{tabular}{|c|c|c|c|c|c|c|c|}
\hline \multirow[t]{2}{*}{ K-value } & \multirow[t]{2}{*}{ Constant } & \multicolumn{6}{|c|}{ Regression coefficient } \\
\hline & & $\mathrm{X}_{1}$ & $\mathrm{X}_{2}$ & $\mathrm{X}_{3}$ & $\mathrm{X}_{4}$ & $\mathrm{X}_{5}$ & $\mathbf{X}_{6}$ \\
\hline 0.0 & 5.1834 & 0.18383 & 0.00537 & -0.00062 & 0.19905 & -0.09925 & 0.00046 \\
\hline 0.1 & 22.2690 & 0.00830 & 0.00399 & 0.00054 & 0.05710 & -0.05345 & 0.00051 \\
\hline 0.2 & 27.8197 & -0.03784 & 0.00269 & 0.00070 & 0.02287 & -0.04909 & 0.00038 \\
\hline 0.3 & 30.4476 & -0.05713 & 0.00230 & 0.00072 & 0.00618 & -0.04830 & 0.00026 \\
\hline 0.4 & 31.8658 & -0.06645 & 0.00205 & 0.00070 & -0.00350 & -0.04793 & 0.00017 \\
\hline 0.5 & 32.6727 & -0.07110 & 0.00186 & 0.00068 & -0.01005 & -0.04752 & 0.00010 \\
\hline 0.6 & 33.1342 & -0.07329 & 0.00172 & 0.00066 & -0.01477 & -0.04702 & 0.00004 \\
\hline 0.7 & 33.3861 & -0.07406 & 0.00161 & 0.00064 & -0.01834 & -0.04643 & -0.00001 \\
\hline 0.8 & 33.5047 & -0.07399 & 0.00151 & 0.00062 & -0.02109 & -0.04580 & -0.00005 \\
\hline 0.9 & 33.5357 & -0.07341 & 0.00143 & 0.00060 & -0.02325 & -0.04512 & -0.00008 \\
\hline 1.0 & 33.5079 & -0.07250 & 0.00058 & 0.00058 & -0.02497 & -0.04443 & -0.00011 \\
\hline
\end{tabular}

Table 6. Comparison between actual and calculated values of serum alanine aminotransferase levels in healthy Chinese adults.

\begin{tabular}{|c|c|c|c|c|c|c|c|c|}
\hline Sample point & $X_{1}$ & $X_{3}$ & $\mathrm{X}_{4}$ & $X_{5}$ & $X_{6}$ & $X_{7}$ & True value & Simulated value \\
\hline Shen Yang & 123.29 & 44.7 & 2574 & 8.4 & 63 & 690.3 & 21.67 & 22.17 \\
\hline Yu Xi & 102.53 & 1636.5 & 2300 & 15.8 & 75 & 906.8 & 29.34 & 26.23 \\
\hline Bao Ding & 115.50 & 85.0 & 2750 & 12.3 & 62 & 570.0 & 21.36 & 22.96 \\
\hline A-K Tao & 75.93 & 1471.0 & 2900 & 11.2 & 48 & 62.8 & 29.34 & 29.30 \\
\hline Guangzhou & 113.22 & 6.3 & 1906 & 21.8 & 79 & 1694.1 & 20.67 & 21.23 \\
\hline Nan Jing & 118.80 & 7.1 & 2155 & 15.3 & 77 & 1062.4 & 21.37 & 21.95 \\
\hline Nan Cong & 106.11 & 309.7 & 1223 & 17.3 & 80 & 987.2 & 21.48 & 21.30 \\
\hline Zun Yi & 106.93 & 860.0 & 1200 & 15.2 & 80 & 1098.0 & 21.35 & 23.10 \\
\hline An Yang & 114.34 & 98.0 & 2400 & 13.6 & 66 & 606.1 & 21.39 & 22.59 \\
\hline Mei Shan & 103.83 & 422.0 & 1060 & 17.2 & 85 & 1057.0 & 23.10 & 21.96 \\
\hline
\end{tabular}




\section{Discussion}

On the condition of hypoxia, sugar in the human body produced pyroracemic acid by the means of glycolysis is turned into alanine under the catalysis of ALT (Wang and Wang, 2010). However, decrease of ALT activity in cold climates leads to a corrective increase of the concentration of this enzyme. Under the colder and drier climate in north-western China, residents have adapted to a propensity for eating food with high fat and protein but with only little fibre content (Jiang and Hu, 2005). The magnitude of fat and protein in this diet is greater than what can be metabolised. On the one hand, the long-term accumulation of lipids in the liver cells increase sensitivity to exogenous damage, while lipids increase the content of bile excretion on the other, a situation which makes cells release excess ALT into serum (Yu et al., 2014). This leads to a change in the internal environment with regional higher levels of ALT as a result.

The effects of soil on ALT values are indirect. Firstly, soil texture has an impact on soil properties in that gravel has better aeration and water permeability, causing a loss of nutrients (Shi et al., 2016) making the soil infertile, as is the case in north-western China. In contrast, the phaiozem soil in north-eastern China has abundant organic matter that produces agriculture products of rich in nutrition (Ling et al., 2016). All else being equal, people in different regions have different diets depending on the local food and this leads to a slight diversity of body fluids and function, e.g., with respect to the need for ALT, which leads to divergent values of the concentration of this enzyme. Secondly, soil pH in China has an evidently regional variation (Yang et al., 2015). The soil on southern China mostly is mostly acid, while alkaline soils are principally clustered in northern China. To balance the $\mathrm{pH}$, people in northern China prefer acidic food, especially people in north-western regions, while people in southern regions dislike acidic food. On this condition, ALT values for healthy adults in north-western regions are higher than those in south-eastern people. Thirdly, the soil cation exchange capacity is an important determinant that to some extent reflects the chemical elements in the soil. Studies have demonstrated that there exists a close link between chemical elements in soil and body fluids that is reflected in a spatial distribution (Wang et al., 2014).

\section{Conclusions}

In summary, the ALT values in healthy adults show significant regional differences in correlation with the ambient geographical environment. In general, the values of this enzyme in the western regions of China are higher than in the East. Knowledge of this is significant for the interpretation of clinical test values and is also important for treatment.
Legend

AFP concerntration (U/L)

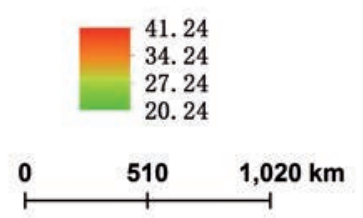

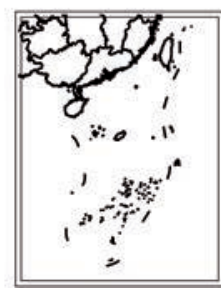

Figure 3. The spatial variation of alanine aminotransferase values in Chinese healthy adults in relation to geography. 


\section{References}

Baksalary JK, Kala R, 1981. Linear transformations preserving best linear unbiased estimators in a general Gauss-Markoff model. Ann Stat 9:913-6.

Bonett DG, Wright, TA, 2000. Sample size requirements for estimating Pearson, Kendall and Spearman correlations. Psychomet 65:23-8.

Drabkin DL, 1975. The environment of function of liver and red blood cells. Ann NY Acad Sci 244:603-23.

Ekiz A, Kaya B, Avci ME, Polat I, Dikmen S, Yildirim G, 2016. Alanine aminotransferase as a predictor of adverse perinatal outcomes in women with intrahepatic cholestasis of pregnancy. Pak J Med Sci 32:418-22.

Fasano G, Franceschini A, 1987. A multidimensional version of the Kolmogorov-Smirnov test. Month Not Roy Astron Soc 50:920.

Ge M, Ranyin X, Jinwei H, Yanyu H, 2014. Geograohic distribution of reference value of boy's peak expiratory flow rate based on the artificial neural networks. Geogr Res 33:451-66.

He XL, Wang HH, 2008. Analysis of Ridge Parameter K. J Lianhe Univ 6:1-3.

Hippokrates, about 400 BC. On airs, waters and places. Available from: http://classics.mit.edu/Hippocrates/airwatpl.html

Hoerl AE, Kennard RW, 1970. Ridge regression: biased estimation for nonorthogonal problems. Technomet 12:55-67.

Jiang L, Hu XJ, 2005. Research and analysis of diet structure and daily activity among the middle and elder People in Lhasa city. J Tibetan Med 24:4-5.

Knudsen AR, Andersen KJ, Hamilton-Dutoit S, Nyengaard JR, Mortensen FV, 2016. Correlation between liver cell necrosis and circulating alanine aminotransferase after ischemia/reperfusion injuries in the rat liver. Int J Exp Pathol 97:133-8.

Ling L, Tao Y, Redden R, 2016. Soil fertility map for food legumes production areas in China. Sci Rep 6:26102.

Liu C, Ge M, Wang C, Lu CA, Yang SF, Cen MY, Zhang W, 2015. The relationship between geographic environments and the reference value of Chinese adults' P-wave dispersion. J Northeast Normal Univ 47:135-40.

Liu HH, Li SJ, 2009. The discussion of ridge regression parameter estimation. J Hainan Univ 27:5-6.

Liu SX, 2015. The clinical significance of liver function test on hepatitis B. Chin J School Doctor 29:938-9.

Lv B, about 200 BC. Available from: http://www.qiuyuge. org/book/34/34621/

Ma L, Cao Y, Xue J, Xu JJ, He JB, 2008. The height of China's 102 population and geographical environment correlation studies. Acta Anthropol Sinica 27:223-30.

Mörl M, 1983. Liver damage caused by occupation and environment. Munch Med Wochenschr 125:40-4.
Rathbun SL, 2012. Interpolation of spatial data: some theory for kriging. Technometrics 95:436-7.

Shi Y, Zhao X, Gao X, Zhang S, Wu P, 2016. The effects of longterm fertilizer applications on soil organic carbon and hydraulic properties of a loess soil in china. Land Degrad Develop 27:60-7.

Song J, Wang K, 2006. Research and analysis of body status between local Tibetans and emigrant han people in Lhasa. Tibetan Med J 27:1-4.

Thakur M, Samanta B, Chakravarty D, 2016. A non-stationary spatial approach to disjunctive kriging in reserve estimation. Spat Stat 17:131-60.

Velleman PF, Welsch RE, 1981. Efficient computing of regression diagnostics. J Amer Stat 35:234-42.

Ušaj A, Burnik S, 2016. The influence of high-altitude acclimatization on ventilatory and blood oxygen saturation responses during normoxic and hypoxic testing. J Hum Kinet 50:125-33.

Wang DZ, 2015. Study on biochemical index in clinical diagnosis of liver cirrhosis. Contemp Med 21:53-4.

Wang H, Mu L, Jiang M, Wang Y, Yan W, Jiao YZ, 2014. The relationship between chemical elements in soil and whole blood, and fluorosis induced by coal-fired pollution. Environ Monit Assess 186:2081-8.

Wang Z, An N, 2010. Research status and progress of alanine aminotransferase. Med J West China 25:239-40.

Wang ZM, Wang WM, 2010. An analysis of the results of health examination of different administrative staff in a plateau. Chin Occup Med 37:433-4.

Watson GS, 1971. Trend-surface analysis. J Int Assoc Math Geol $3: 215-26$.

Xiao L, 2014. Clinical value analysis of six liver function indexes in the diagnosis of liver diseases. Contemp Med Forum 12:42-3.

Xue J, 2014. Six Items Liver function index of the joint test in liver disease diagnosis of clinical value analysis. Chin J Geriatric Care 12:104-5.

Yang N, 2004. The unique role of ridge regression analysis in solving the problem of multiple collinearity. Stat Dec 3:14-5.

Yang Y, Li P, He H, Zhao X, Datta A, ma W, Zhang Y, Liu X, Han WC, Wilson M, Fang J, 2015. Long-term changes in soil PH across major forest ecosystems in China. Geophys Res Lett 42:933-40.

Yu CH, 2007. Analysis of SPSS and statistics. Electronic industry press, Beijing, China.

Yu XY, Zhao Y, Song XX, Song ZY, 2014. The association between body mass index and non-alcoholic fatty liver disease. J Zhejiang Univ 4:546-52.

Zar JH, 1972. Significance testing of the spearman rank correlation coefficient. J Am Stat Assoc 67:578-80.

Zhu HB, He LJ, 2009. A study on appropriate conditions in consistency test of normal distribution by Single Sample K-S Check in SPSS. J Capit Inst Phys Edu 21:466-70. 